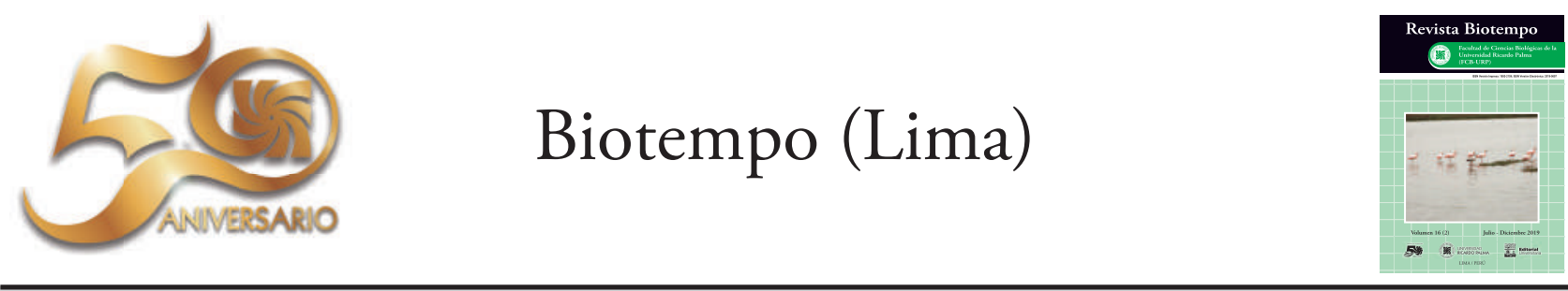

ORIGINAL ARTICLE / ARTÍCULO ORIGINAL

SPATIAL AND TEMPORAL DISTRIBUTION OF BIRDLIFE AT THE INTERNATIONAL AIRPORT JORGE CHÁVEZ CALLAO-PERÚ

\title{
DISTRIBUCIÓN ESPACIAL Y TEMPORAL DE AVIFAUNA EN EL AEROPUERTO INTERNACIONAL JORGE CHÁVEZ, CALLAO-PERÚ
}

\author{
Carlos Alberto Elías-Cruzado ${ }^{1}$ \& Flor de María Madrid-Ibarra ${ }^{1}$
}

1 Instituto de Recursos Naturales y Ecología (IRNE). Facultad de Ciencias Biológicas Universidad Ricardo Palma Lima-Perú.

calielias2@gmail.com; flordemaria.madrid@urp.edu.pe

Author for correspondence: flordemaria.madrid@urp.edu.pe

\begin{abstract}
Around airports, there are external conditions that turn out to be attractive for birds that include wetlands, grasslands, crops, mangroves, organic waste from human activities, and taxiways for aircraft which are permanent source for bird transit. Jorge Chávez International Airport (AIJCh) located in Lima, Peru presents many of the above mentioned factors, so the evaluation of avian fauna is necessary. The objective of this work is to perform the spatial and temporal evaluation through surveys between November 2015 and July 2016 and use the Avian Risk Assessment Index (IERA in Spanish) to detect those species that generate the greatest risk. The groups of species that presented greater abundance were the gulls (Family Laridae) with a maximum reported of 5189 individuals and the "black vulture" (Coragyps atratus (Bechstein, 1793) with a maximum reported of 371 individuals. Both groups were more active from the first hours of the morning $(9: 00 \mathrm{am})$ and in the mid-afternoon $(4: 00 \mathrm{pm}$ to 5:00 pm). In addition, these groups also presented higher risk according to the IERA. This index works efficiently as a diagnostic tool for potentially dangerous bird species for air navigation.
\end{abstract}

Keywords: airports - avian risk - spatial and temporal distribution

\section{RESUMEN}

Alrededor de los aeropuertos se encuentran condiciones externas que resultan ser atrayentes para las aves las cuales incluyen: humedales, pastizales, sembríos, manglares, desmontes de residuos orgánicos provenientes de actividades antrópicas, además las pistas de rodaje para las aeronaves son foco de tránsito permanente de aves. El aeropuerto Internacional Jorge Chávez (AIJCh) ubicado en Lima, Perú presenta muchos de los factores mencionados por lo que la evaluación de la fauna aviar es necesaria. El objetivo de este trabajo es realizar la evaluación espacial y temporal mediante censos por punto de conteo durante noviembre del 2015 hasta julio 2016 y utilizar el Índice de Evaluación de Riesgo Aviario (IERA) para detectar aquellas que generan riesgo. Las especies que presentaron mayor abundancia fueron las gaviotas (Familia Laridae) con un máximo reportado de 5189 individuos y los gallinazos (Coragyps atratus (Bechstein, 1793)) 
con un máximo registrado de 371 individuos. Ambos grupos estuvieron más activos a partir de las primeras horas de la mańana $(9: 00 \mathrm{~h})$ y en la media tarde $(16: 00-17: 00 \mathrm{~h})$. Estos grupos también presentaron mayor riesgo según el IERA. Dicho índice funciona eficientemente como herramienta de diagnóstico de especies de aves potencialmente peligrosas.

Palabras clave: aeropuerto - distribución espacial y temporal - peligro aviario

\section{INTRODUCCIÓN}

La presencia de la población de aves que eventualmente o permanentemente se encuentran dentro de las áreas operativas de un aeropuerto responde a diferentes factores, generalmente suelen ser atraídas por condiciones tan esenciales como el alimento, el agua, un lugar de abrigo o descanso, las migraciones, el clima, el tránsito aéreo, los tipos de aeronaves e incluso la misma infraestructura del aeropuerto pueden ofrecer zonas de anidamiento, alimentación y percha (Sodhi, 2002; Leoncio et al., 2010).

Alrededor de los aeropuertos se encuentran condiciones externas que resultan ser atrayentes para las aves las cuales incluyen: humedales, pastizales, sembríos, manglares, desmontes de residuos orgánicos provenientes de actividades antrópicas, además las pistas de rodaje para las aeronaves son foco de transito permanente de aves (Mendonça, 2009; Ayala-Pérez et al., 2013; Ning \& Chen, 2014).

Los factores que afectan la distribución y abundancia de las aves son la temporalidad, las estaciones del año y algunas variables ambientales como la temperatura, la distribución espacial de los recursos alimenticios, el tipo de sustrato, la comunidad vegetal y sus tiempos de alimentación. Además, el viento afecta la distribución y abundancia ya que en días de fuertes vientos las aves prefieren sitios menos expuestos como los hangares o las estructuras de los puentes de embarque (Guzmán et al., 1994; González \& Málaga, 1997).

Aunque la colisión de un ave con una aeronave no cause pérdidas humanas, en la mayoría de los casos se producen daños materiales en partes de la aeronave como en los motores, fuselaje, alas (alerones y flaps), tren de aterrizaje y, en menor medida, la cola (Linnell et al., 1999; Barras \& Dolbeer, 2000; Owino et al., 2004). Por consiguiente organismos internacionales reconocen la importancia de poner en funcionamiento planes de manejo en aquellos aeropuertos donde el riesgo aviar sea un factor importante (Ning \& Chen, 2014; Meer, 2019). Por ello un programa de control y monitoreo exitoso debe resultar de evaluaciones específicamente realizadas para cada sitio particular (Novaes \& Alvarez, 2010; Madrid \& Elías, 2017).
El aeropuerto Internacional Jorge Chávez (AIJCh) presenta muchos de los factores antes mencionados por lo que la evaluación de la distribución espacial y temporal es necesaria para el establecimiento de un plan de monitoreo aviar (Ralph et al., 1993; Reca et al., 1994; Bibby et al., 1998; Gatto et al., 2005; Marateo et al., 2011). El AIJCh, se encuentra ubicado en la Provincia Constitucional del Callao, a $10 \mathrm{~km}$ del centro de Lima, limitando al Norte y Oeste por zonas fragmentadas, antes humedales, al Sur por el rio Rímac y al Este por un ecosistema urbano, siendo el objetivo general de esta investigación determinar la distribución espacial y temporal de Avifauna en el Aeropuerto Internacional Jorge Chávez, Callao-Perú.

\section{MATERIALES Y METODOS}

\section{Área de estudio}

El proyecto se realizó, en base a los datos obtenidos por el investigador responsable en el Aeropuerto Internacional Jorge Chávez (AIJCh), ubicado en la Avenida Elmer Faucett $s / n$, provincia constitucional del Callao y departamento de Lima, durante los meses de octubre 2015 a julio 2016 (Fig. 1).

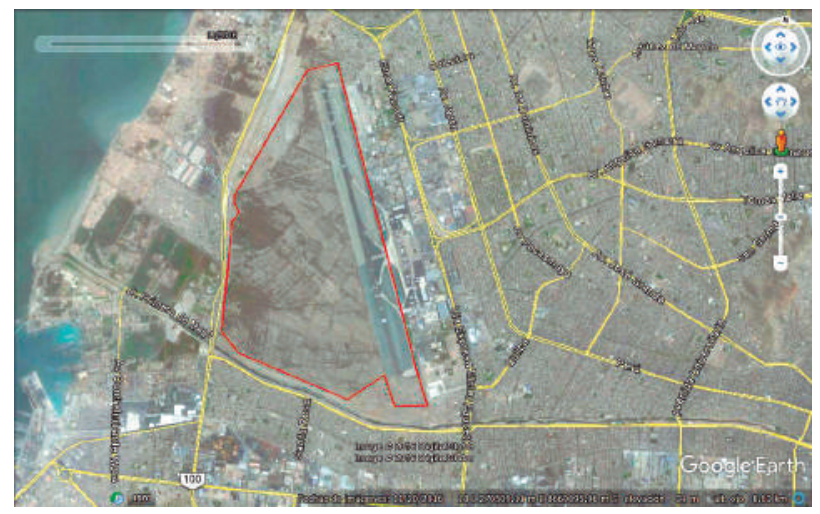

Figura 1. Delimitación de la zona de estudio.

\section{Variables}

En el desarrollo de la investigación se analizaron 8 variables que son el resultado de las acciones tomadas para la ejecución de los objetivos planteados. Variables 
dependientes: Abundancia, tamańo corporal, grado de agregación, uso del espacio vertical, uso de sector, uso del hábitat, riesgo de uso de zonas y antecedentes de incidentes.

Tabla 1. Operacionalización de variables, distribución espacial y temporal de avifauna en el Aeropuerto Internacional Jorge Chávez, Callao-Perú.

Variable

\begin{tabular}{|c|c|c|c|c|c|}
\hline Tipo & Nombre & Definición & Naturaleza & Indicador & Referencia \\
\hline \multirow{26}{*}{ 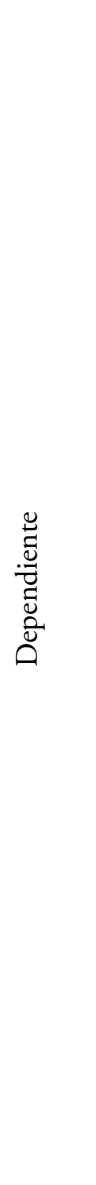 } & Abundancia & Número de individuos por especie. Los & Cuantitativa & Número de & Reca et al.(1994) \\
\hline & & límites entre las categorías de abundancia & & individuos & \\
\hline & & serán establecidos por medio del cálculo & & & \\
\hline & & promedio y desviación estándar. & & & \\
\hline & Tamaño corporal & Masa corporal de aves obtenida a través de & Cuantitativa & Masa en gramos & Schulenberg et al. \\
\hline & & literatura & & & $(2010)$ \\
\hline & Grado de agregación & Conteo de individuos por hábitos gregarios & Cuantitativa & Grupos según & Marateo et al. \\
\hline & & y que se muevan en bandadas. & & número de & $(2011)$ \\
\hline & & & & individuos y & \\
\hline & & & & dispersión & \\
\hline & Uso del espacio & Los avistamientos se remitirán a 3 & Cualitativa & Número de & Marateo et al. \\
\hline & vertical & categorías de altura: a nivel de suelo, hasta & & individuos & (2011) \\
\hline & & $50 \mathrm{~m}$ y a más de $50 \mathrm{~m}$ de altura. & & & \\
\hline & Uso del sector & Sectores del aeropuerto donde se & Cualitativa & Número de & Reca et al. (1994) \\
\hline & & encuentran especies de aves & & individuos por & \\
\hline & & & & sector. & \\
\hline & Uso del hábitat & Hábitats dentro del aeropuerto donde se & Cualitativa & Número de & Reca et al. (1994) \\
\hline & & encuentran especies de aves & & individuos por & \\
\hline & & & & hábitat & \\
\hline & Riesgo de uso de & En función del resigo potencial, se verifica & Cualitativa & Número de & Marateo et al. \\
\hline & zonas & la presencia de aves en ciertas zonas. & & individuos por & $(2011)$ \\
\hline & & & & zonas de riesgo & \\
\hline & Antecedentes de & Aquellas especies que hayan sido & Cualitativa & Reportes de & Marateo et al. \\
\hline & incidentes & reportadas, de cualquier forma, o a través & & impacto dentro o & $(2011)$ \\
\hline & & de cualquier medio, como intervinientes en & & fuera de la zona de & \\
\hline & & un incidente o accidente aéreo & & la estudio & \\
\hline
\end{tabular}

\section{Evaluación cuantitativa}

Para la evaluación de la estructura de la comunidad de avifauna, la riqueza de especies y abundancia de las poblaciones, se empleó el censo por conteo de puntos fijos, método a través del cual el observador permanece en un punto fijo, y registra las aves vistas y escuchadas en un tiempo y radio determinado Se consideraron 3 estaciones de muestreo con sus respectivos puntos de conteo (15) en donde se registró el número de individuos observados durante un tiempo aproximado de $10 \mathrm{~min}$, dejando transcurrir un minuto luego de la llegada del observador al punto de evaluación, con el fin de disminuir el disturbio y detectar la mayor cantidad de aves posibles. Las especies observadas e identificadas fueron fotografiadas (Bibby et al., 1998). La intensidad de muestreo y la delimitación de áreas se estableció en campo, tomando en consideración el tamaño del área de evaluación y los hábitats presentes, de manera que el esfuerzo de muestreo realizado sea representativo, tanto estadística como ecológicamente para la zona (Ralph et al.,1993). En este sentido los puntos establecidos para cada zona fueron distribuidos de la siguiente forma: 3 puntos de conteo para la Cabecera 33 (Fig. 2), 2 puntos de conteo para la Cabecera 15 (Fig. 3) y 10 puntos de conteo para chacra Oeste (Fig. 4), espacios evaluados en el AIJCH. 


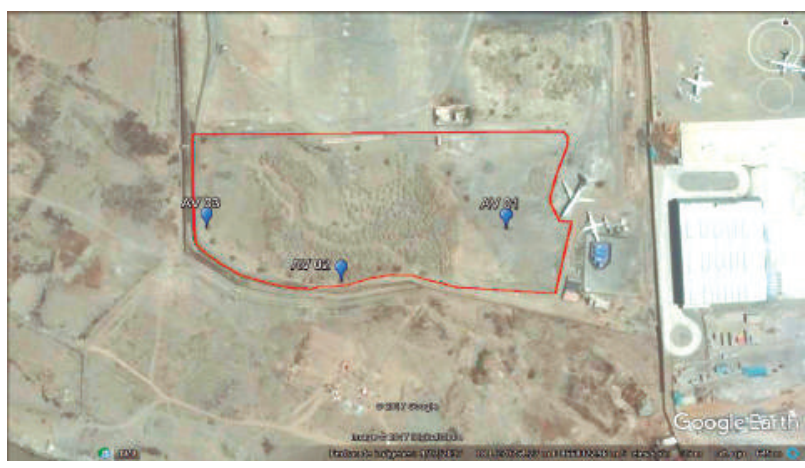

Figura 2. Delimitación de la zona de Cabecera 33 con los puntos conteo establecidos.

Tabla 2. Coordenadas UTM de los puntos de conteo de la zona de Cabecera 33.

\begin{tabular}{ccc}
\hline Punto de conteo & \multicolumn{2}{c}{ Coordenadas UTM } \\
& Este & Norte \\
\hline AV 01 & 270842 & 8668359 \\
AV 02 & 270739 & 8668283 \\
AV 03 & 270588 & 8668288 \\
\hline
\end{tabular}

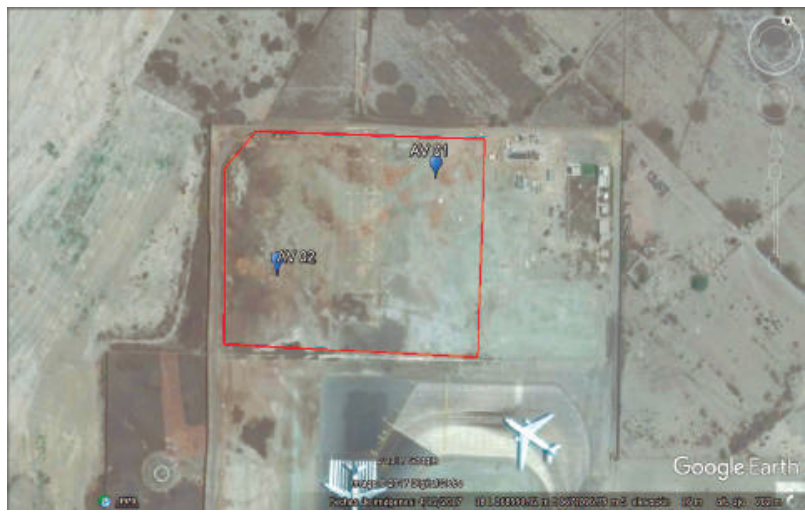

Figura 3. Delimitación de la zona de Cabecera 15 con los puntos conteo establecidos.

Tabla 3. Coordenadas UTM de los puntos de conteo de la zona de Cabecera 15.

\begin{tabular}{ccc}
\hline \multirow{2}{*}{ Punto de conteo } & \multicolumn{2}{c}{ Coordenadas UTM } \\
& Este & Norte \\
\hline AV 01 & 269004 & 8671993 \\
AV 02 & 268847 & 8671824 \\
\hline
\end{tabular}

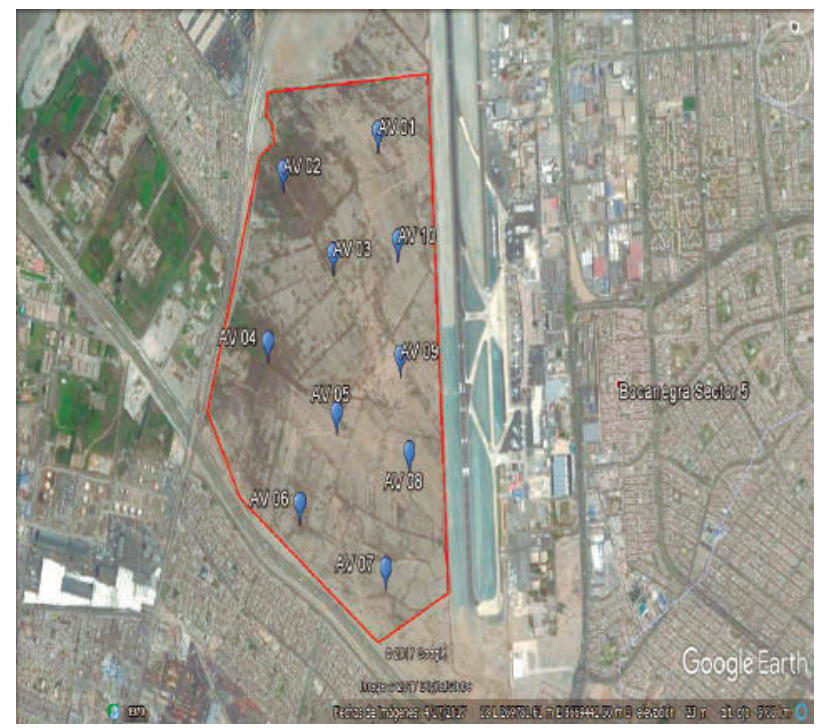

Figura 4. Delimitación de la zona de Chacra Oeste con los puntos conteo establecidos.

Tabla 4. Coordenadas UTM de los puntos de conteo de la zona de Chacra oeste.

\begin{tabular}{ccc}
\hline \multirow{2}{*}{ Punto de conteo } & \multicolumn{2}{c}{ Coordenadas UTM } \\
& Este & Norte \\
\hline AV 01 & 268937 & 8670434 \\
AV 02 & 268476 & 8670254 \\
AV 03 & 268616 & 8669760 \\
AV 04 & 268692 & 8669126 \\
AV 05 & 268965 & 8668515 \\
AV 06 & 269315 & 8668888 \\
AV 07 & 269978 & 8668510 \\
AV 08 & 269882 & 8668958 \\
AV 09 & 269546 & 8669573 \\
AV 10 & 269269 & 8669939 \\
\hline
\end{tabular}

\section{Determinación de especies}

Para la determinación de especies se utilizó la edición en castellano de la guía de campo "Aves de Perú" (Schulenberg et al., 2010). No se realizaron colectas.

De manera adicional para la actualización de nombres científicos y taxonomía se utilizó la lista del comité de clasificación de Sudamérica (SACC por sus siglas en inglés) (Remsen, 2016) y la base datos xeno-canto para identificación de sonidos (Xenacanto 2019). 
Índice de evaluación de riesgo aeronáutico (IERA)

El IERA se utilizó para conocer el grado de riesgo aviar para las operaciones aeronáuticas, considerando que un alto valor corresponde a un mayor nivel de riesgo y un valor más bajo se asocia a niveles menores de riesgo. Se definen 8 variables:

\section{1.- Abundancia (Abu)}

Se asumió que la especie más común o frecuente representara un riesgo mayor que una más rara. Los límites entre las categorías de abundancia serán establecidos por medio del cálculo promedio y desviación estándar de las abundancias relativas que se obtendrán por los muestreos de campo.

0: rara. Corresponde a todas las especies con un valor de abundancia relativa menor al promedio

1: común. Corresponde a todas las especies con un valor de abundancia relativa ubicado entre el promedio y el promedio más la desviación estándar

2: abundante. Corresponde a todas las especies con un valor de abundancia mayor al promedio más la desviación estándar

\section{2.-Tamańo Corporal (Tam)}

Se asume que un ave de mayor tamańo representa un riesgo mayor para las aeronaves que una de menor tamańo.

0: menos de $100 \mathrm{~g}$ de masa corporal

1: entre 100 y $500 \mathrm{~g}$ de masa corporal

2: más de $500 \mathrm{~g}$ de masa corporal

\section{3.- Grado De Agregación (Agr)}

Se asume que aquellas especies que tengan hábitos gregarios y se muevan en bandadas son más peligrosas que las que no tienen este comportamiento.

0 : Solitarios, en parejas o en grupos de menos de 5 individuos.

1: grupos de entre 5 y 50 individuos dispersos.

2: grupos de entre 5 y 50 individuos compactos.

3: grupos de más de 50 individuos

\section{4.- Uso Del Espacio Vertical (Vert)}

Los avistamientos se remitieron a 3 categorías de altura: a nivel de suelo, hasta $50 \mathrm{~m}$ y a más de $50 \mathrm{~m}$ de altura.
Se asume que aquellas aves que utilizan generalmente un único estrato vertical son menos peligrosas que las que hacen generalmente uso de más de uno.

0: usa generalmente un único estrato

1: usa generalmente dos estratos

2: eso generalmente los 3 estratos

\section{5.- Uso De Sector (Sect)}

Se asume que aquellas aves que se encontraron en un único sector son menos peligrosas para la aeronavegación que las que se encontraron en más de uno.

0: usa un único sector

1: uso dos o tres sectores

2: uso cuatro o más sectores

\section{6.- Uso Del Hábitat (Hab)}

Se asume que aquellas especies que aprovechan varios tipos de hábitats que son potencialmente más peligrosas que las que se relacionan a un único hábitat.

0: usa un único hábitat

1: usa dos hábitats

2: usa tres o más hábitats

\section{7.- Riesgo de impacto por uso De Zonas (Ruzon)}

En función del resigo potencial que puede representar la presencia de las aves en ciertas zonas.

0 : Se vio en las zonas aledañas de la pista

1: Se vio en la pista

2: se vio en las zonas de despegue y/o aterrizaje

\section{8.-Antecedentes De Incidentes (Inci)}

Se asumirá aquellas especies que hayan sido reportadas como intervinientes en un incidente o accidente aéreo.

0: no existen antecedentes de ningún tipo

1: existen antecedentes, referencia de otras áreas fuera del AIJCh

2: existen antecedentes, referencia en zonas dentro del AIJCh

\section{ANÁLISIS DE DIVERSIDAD}

Con la finalidad de evaluar la diversidad alfa (Biodiversidad de Simpson 1-D e índice de Shannon Wiener) se utilizó el software PAST ver 3,14 y finalmente Microsoft Excel 
2017 para el procesamiento de cuadros y operaciones básicas. De esta manera se presentan las tablas de resultados correspondientes, estimando los parámetros descriptivos y para los cálculos respectivos.

\section{Aspectos éticos}

Los autores señalan que se cumplieron todas las normas éticas nacionales e internacionales.

\section{RESULTADOS Y DISCUSIÓN}

En el aeropuerto internacional Jorge Chávez, durante los 9 meses de observación de noviembre del 2015 a julio del 2016, se registraron un total de 31 especies de aves, predominando especies residentes, salvo una especie migratoria la cual corresponde a Leucophaeus pipixcan (Wagler, 1831). La riqueza específica de las zonas monitoreadas presentó pocas variaciones a lo largo de la duración del estudio, con mayor riqueza en el mes de noviembre 2015 con un total de 28 especies registradas y menor riqueza en los meses de febrero a junio 2016 con 20 especies registradas. Durante los 9 meses de observación en el mes de abril se obtuvo la mayor abundancia para las 3 zonas que fue de 6542 individuos, seguido por el mes de marzo con 3784 y en tercer lugar los meses de diciembre y enero, ambos con una suma de 857 individuos para las 3 zonas (Fig. 4).

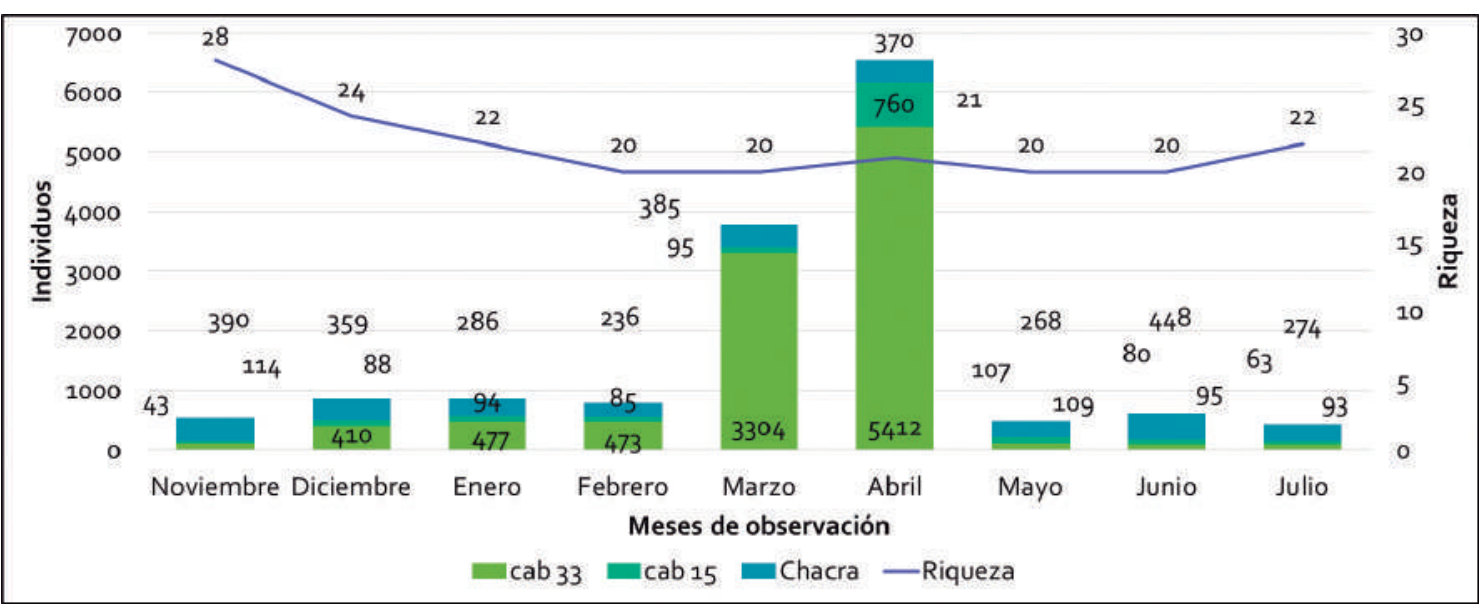

Figura 4. Riqueza específica y abundancia temporal. La abundancia temporal fue dividida considerando tres zonas del AIJCh. Cabecera 33 (cab 33) Cabecera 15 (cab 15) y Chacra Oeste (Chacra).

La comunidad estuvo notoriamente dominada por las especies de la familia Laridae (Larus belcheri, Vigors, 1829, Larus dominicanus, Lichtenstein, 1823 y Leucophaeuspipixcan (Wagler 1831) dicha familia será considera dentro del análisis de abundancia como si de una especie se tratase debido al alto pico de actividad que presenta. La segunda especie más abundante fue Coragyps atratus (Bechstein, 1793) con una abundancia máxima de 371 individuos registrados, Los miembros de la familia Columbidae: Zenaida auriculata (Des
Murs, 1847) (LC) , Zenaida meloda (Tschudi 1843) (LC) y Columba livia (Gmelin, 1789) (LC) se reportaron de manera constante en las zonas de Cabecera 33 y Cabecera 15, su presencia está relacionada con las modificaciones del paisaje y el tipo de hábitat que son factores clave para determinar la presencia en los aeropuertos. (Kuster, 2000; Washburn \& Seamans, 2004) lo que concuerda también con Madrid \& Elías (2017) donde estas especies fueron observadas en ecosistemas urbanos. 
Tabla 5. Especies de aves observadas durante el tiempo de duración del estudio.

\begin{tabular}{|c|c|c|}
\hline Especies & $\begin{array}{c}\text { Registros } \\
\text { totales }\end{array}$ & $\begin{array}{l}\text { Conteo } \\
\text { máximo } \\
\end{array}$ \\
\hline Amazilia amazilia (Lesson,1827) & 19 & 3 \\
\hline Athene cunicularia (Molina, 1782) & 15 & 3 \\
\hline Bulbucus ibis (Linnaeus, 1758) & 10 & 4 \\
\hline Burhinus superciliaris (Tschudi,1843) & 21 & 6 \\
\hline Camptostoma obsoletum (Temminck, 1824) & 33 & 7 \\
\hline Charadrius vociferus (Linnaeus, 1758) & 4 & 3 \\
\hline Chordeiles acutipennis Hermann, 1783) & 10 & 5 \\
\hline Columba livia (Gmelin, 1789) & 107 & 17 \\
\hline Columbina cruziana (Prévost, 1842) & 39 & 8 \\
\hline Coragyps atratus (Bechstein,1793) & 4007 & 371 \\
\hline Crotophaga sulcirostris (Swainson, 1827) & 121 & 19 \\
\hline Falco peregrinus (Tunstall, 1711) & 54 & 6 \\
\hline Falco sparverius (Linnaeus, 1758) & 30 & 4 \\
\hline \multicolumn{3}{|l|}{ Larus belcheri (Vigors,1829) } \\
\hline Larus dominicanus (Lichtenstein, 1823) & 9384 & 5189 \\
\hline \multicolumn{3}{|l|}{ Leuphaeus pipixcan (Wagler, 1831) } \\
\hline Parabuteo unicintus (Temminck, 1824) & 48 & 9 \\
\hline Passer domesticus (Linnaeus, 1758) & 28 & 7 \\
\hline Pygochelidion cyanoleuca (Vieillot, 1817) & 89 & 27 \\
\hline Pyrocephalus rubinus (Boddaert, 1783) & 13 & 2 \\
\hline Sicalis luteola (Sparrman, 1789) & 291 & 127 \\
\hline Sporophila simplex (Taczanowski, 1874) & 56 & 16 \\
\hline Sporophila telasco (Lesson, 1828) & 118 & 20 \\
\hline Thinocorus rumicivorus (Eschscholtz, 1829) & 2 & 1 \\
\hline Troglodytes aedon (Vieillot, 1809) & 61 & 13 \\
\hline Tyrannus melancholicus (Vieillot, 1819) & 2 & 1 \\
\hline Tyto alba (Scopoli, 1769) & 1 & 1 \\
\hline Volatinia Jacarina (Linnaeus, 1766) & 54 & 21 \\
\hline Zenaida auriculata (Des murs, 1847) & 88 & 15 \\
\hline Zenida meloda (Tschudi, 1843) & 157 & 17 \\
\hline Zonotrichia capensis (Statius Müller, 1776) & 23 & 8 \\
\hline Riqueza: 31 & 14885 & 5930 \\
\hline
\end{tabular}



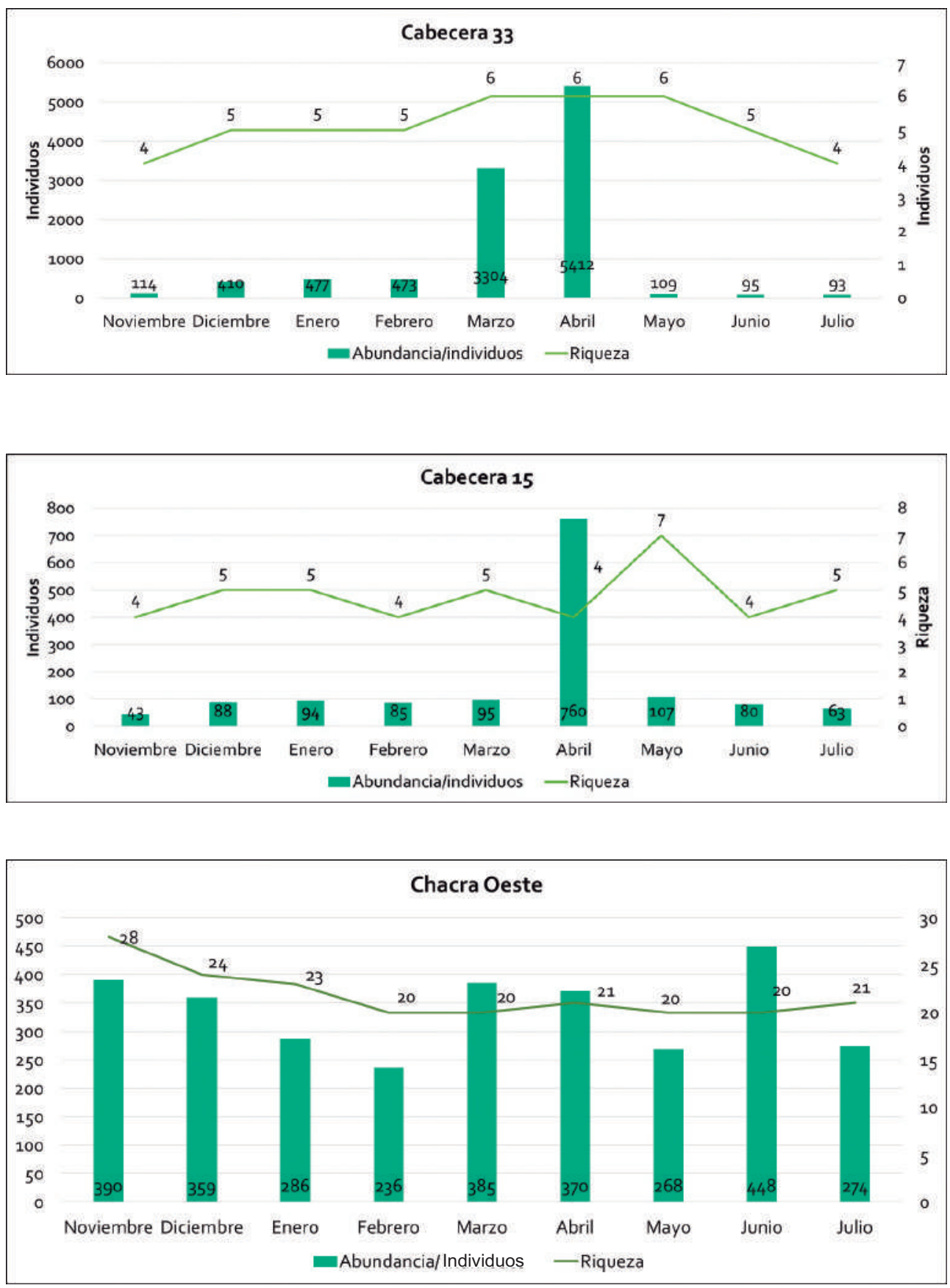

Figura 5. Riqueza específica y abundancia temporal de las zonas: Cabecera 33; Cabecera 15 y Chacra oeste, espacios correspondientes al Aeropuerto Internacional Jorge Chávez

La figura 5 representa de manera comparativa el núnero de especies y el total de individuos en las tres zonas evaluadas, siendo la chacra oeste lugar donde se registró la mayor cantidad de especies sin embargo tanto el tamaño corporal de las aves, como el riesgo de uso de zonas, no representan riesgo potencial durante los meses de observación.
En cuanto al análisis de abundancia si se presentaron oscilaciones sumamente marcadas entre las 3 zonas, siendo los meses de marzo y abril del 2016 aquellos donde se registró el mayor pico de abundancia caracterizándose por el tránsito de miles de individuos de la familia Laridae en su mayoría pertenecientes a la especie L. pipixcan, especie migratoria y, concordando con Villegas \& Herrera (2014) quienes monitorearon el movimiento migratorio de estas 
especies y registraron el número de aves, la altura a la que volaron y los factores ambientales como la dirección del viento, se reportaron individuos volando a menos de 80 $m$ en donde es probable que ocurra una colisión

\section{Clasificación de la avifauna según su riesgo potencial para la aeronavegación}

Se clasificaron las especies y/o grupos de especies de acuerdo al grado de riesgo potencial para la aeronavegación. En la presente investigación se presenta un sistema de categorización que se basa en una serie de atributos o variables propias de cada una de las especies, el cual es denominado Índice de Evaluación de Riesgo aeronáutico (IERA). Dichos atributos corresponden a: abundancia, tamaño corporal, grado de agregación, uso de espacio vertical, uso de sector, uso de hábitat, Riesgo de impacto por uso de zonas y antecedentes de incidente.

Tabla 6. Lista de especies de aves registradas en el AIJCh, indicando el valor que adquiere para cada variable y el valor final del IERA siendo este criterio por el cual se organizan.

\begin{tabular}{cccccccccc}
\hline Especies & Abu & Tam & Agr & Vert & Sect & Hab & Ruzon & Inci & IERA \\
Coragyps atratus & 2 & 2 & 3 & 2 & 2 & 2 & 2 & 1 & 16 \\
Leucophaeus pipixcan & 2 & 1 & 3 & 2 & 2 & 1 & 2 & 1 & 14 \\
Larus belcheri & 1 & 2 & 2 & 2 & 2 & 1 & 2 & 1 & 13 \\
Larus dominicanus & 1 & 2 & 2 & 2 & 2 & 1 & 2 & 1 & 13 \\
Falco peregrinus & 1 & 2 & 0 & 2 & 2 & 1 & 2 & 0 & 10 \\
Parabuteo unicintus & 1 & 2 & 0 & 2 & 2 & 1 & 2 & 0 & 10 \\
Zenaida auriculata & 1 & 1 & 1 & 1 & 2 & 2 & 1 & 0 & 9 \\
Zenida meloda & 1 & 1 & 1 & 1 & 2 & 2 & 1 & 0 & 9 \\
Columba livia & 1 & 1 & 1 & 1 & 1 & 2 & 1 & 0 & 8 \\
Sicalis luteola & 2 & 0 & 3 & 1 & 0 & 0 & 0 & 0 & 6 \\
Falco sparverius & 0 & 1 & 0 & 1 & 1 & 1 & 1 & 0 & 5 \\
Burhinus superciliaris & 1 & 2 & 0 & 0 & 0 & 0 & 0 & 0 & 3 \\
Columbina cruziana & 1 & 0 & 0 & 1 & 0 & 1 & 0 & 0 & 3 \\
Passer domesticus & 1 & 0 & 1 & 1 & 0 & 0 & 0 & 0 & 3 \\
Pygochelidion cyanoleuca & 1 & 0 & 1 & 1 & 0 & 0 & 0 & 0 & 3 \\
Tyto alba & 0 & 2 & 0 & 1 & 0 & 0 & 0 & 0 & 3 \\
Athene cunicularia & 1 & 1 & 0 & 0 & 0 & 0 & 0 & 0 & 2 \\
Bulbucus ibis & 1 & 1 & 0 & 0 & 0 & 0 & 0 & 0 & 2 \\
Charadrius vociferus & 1 & 1 & 0 & 0 & 0 & 0 & 0 & 0 & 2 \\
Crotophaga sulcirostris & 1 & 0 & 0 & 1 & 0 & 0 & 0 & 0 & 2 \\
Pyrocephalus rubinus & 1 & 0 & 0 & 1 & 0 & 0 & 0 & 0 & 2 \\
Sporophila simplex & 1 & 0 & 0 & 1 & 0 & 0 & 0 & 0 & 2 \\
Sporophila telasco & 1 & 0 & 0 & 1 & 0 & 0 & 0 & 0 & 2 \\
Troglodytes aedon & 1 & 0 & 0 & 1 & 0 & 0 & 0 & 0 & 2 \\
Volatinia Jacarina & 1 & 0 & 0 & 1 & 0 & 0 & 0 & 0 & 2 \\
Zonotrichia capensis & 1 & 0 & 0 & 1 & 0 & 0 & 0 & 0 & 2 \\
Camptostoma obsoletum & 1 & 0 & 0 & 0 & 0 & 0 & 0 & 0 & 1 \\
chordeiles acutipennis & 1 & 0 & 0 & 0 & 0 & 0 & 0 & 0 & 1 \\
Thinocorus rumicivorus & 0 & 0 & 0 & 1 & 0 & 0 & 0 & 0 & 1 \\
Tyrannus melancholicus & 0 & 0 & 0 & 1 & 0 & 0 & 0 & 0 & 1 \\
Amazilia amazilia & 0 & 0 & 0 & 0 & 0 & 0 & 0 & 0 & 0 \\
\hline & & & & & & & & \\
\hline
\end{tabular}

(Abu=Abundancia; Tam=Tamaño Corporal; Agr=Grado de agregación; Vert =Uso Del Espacio Vertical; Sect=Uso De Sector; Hab= Uso Del Hábitat; Ruzon= Riesgo de impacto por uso De Zonas; Inci = Antecedentes De Incidentes). 
De acuerdo con los valores del IERA, la especie $C$. atratus resultó ser potencialmente la más peligrosa para las operaciones aéreas en el AIJCh con 16 puntos, En segundo lugar, se encuentra un miembro de familia Laridae que corresponde a L. pipixcan con 14 puntos, igualmente que la especie anterior el máximo puntaje que obtuvo fue de 3 en grado de agregación puesto que se registraron miles de individuos. El resto de los miembros de la familia Laridae (L. belcheri y L. dominicanus obtuvieron 13 puntos cada uno, si bien tienen características similares con $L$. pipixcan, la diferencia de puntajes se debe a que presentan una menor abundancia que la especie anterior,

En cuanto a las especies Falco peregrinus (Tunstall, 1711) y Parabuteo unicinctus (Temminck, 1824) ambos obtuvieron 10 puntos, no presentan una abundancia significativa, el tamaño corporal, la altura de vuelo que utilizan y su presencia constante en la zona eleva el índice de riesgo por lo que se consideran especies de riesgo. El resto de especies obtuvieron un valor menor a 10 puntos, por lo que no se consideran especies potencialmente peligrosas.

La ubicación geográfica del Aeropuerto Jorge Chávez, el aumento del tráfico aéreo y si el hombre continúa invadiendo el hábitat natural, continuarán agravándose los problemas de colisión de aves (Dukija \& Gahlot, 2013).

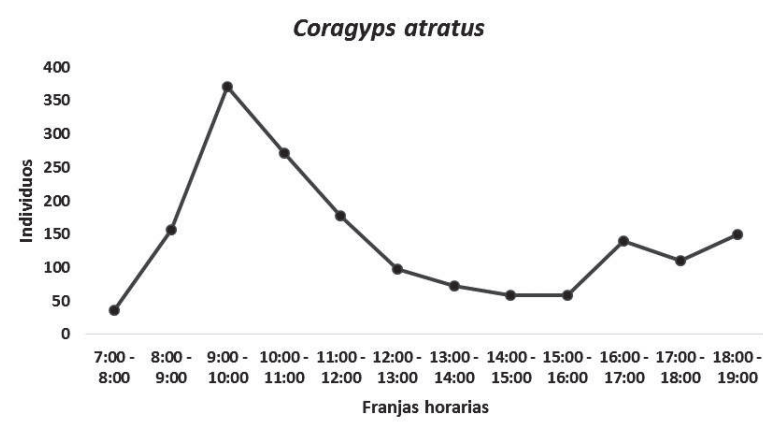

Figura 7. Periodo de actividad de Coragyps atratus.

Coragyps atratus comienza con un periodo de actividad bajo en la franja horaria de 7:00-8:00 h con 36 individuos, este número fue incrementándose hasta llegar a una abundancia máxima de 371 individuos registrados entre las 9:00-10:00 h luego disminuye paulatinamente hacia las 14:00-15:00 h con 59 individuos registrados en dicha franja horaria. Finalmente, se presenta un ligero incremento con 139; 110 y 150 individuos entre las 16:00 y 19:00 h respectivamente (Fig. 7).

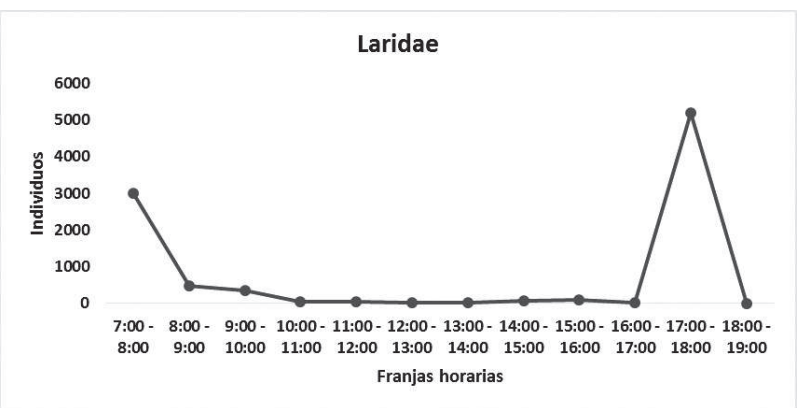

Figura 8. Periodo de actividad de la familia Laridae.

La familia Laridae tuvo un registro de actividad con una abundancia de 3000 individuos entre las 7:00- 8:00 h, la que disminuyó durante el día, para luego llegar a la abundancia máxima registrada de 5189 individuos entre las 17:00-18:00 h (Fig. 8).

\section{Análisis de biodiversidad}

Para las 3 zonas se evaluaron los siguientes índices de biodiversidad alfa: Diversidad de Simpson 1-D e Índice de Shannon-Wiener (Fig. 9).

Cabecera 33 la diversidad de Simpson 1-D tuvo un valor mínimo de 0,079 bits/ind y un valor máximo de 0.473 bits/ind lo que sugiere que dentro de la zona hay una especie dominante, corroborado con los avistamientos de C. atratus que en varios casos superan los 300 individuos. El índice de Shannon-Wiener obtuvo un valor mínimo 0,183 bits/ind y un valor máximo de 0,992 bits/ind. Lo cual indica una baja biodiversidad para esta zona dentro del AIJCh

Cabecera 15 En esta zona la diversidad de Simpson 1-D tuvo variaciones marcadas a lo largo de los meses de estudio y se explica por los cambios en la riqueza y número de individuos que fue mucho menor con respecto a la Cabecera 33. El índice de Shannon-Wiener presentó un valor mínimo de 0,411 bits/ind y un máximo de 1,133 bits/ind dando como resultado una biodiversidad baja para la zona.

Chacra oeste A diferencia de las otras zonas tuvo valores más altos para la diversidad de Simpson D y de Shannon-Wiener. Dichos índices se vieron afectados en el mes de abril donde presentaron sus valores más bajos Para el índice de Shanon-Wiener se obtuvieron valores que van desde un mínimo de 1,378 bits/ind hasta un valor máximo de 2,561 bits/ind, lo que sugiere una biodiversidad intermedia. 

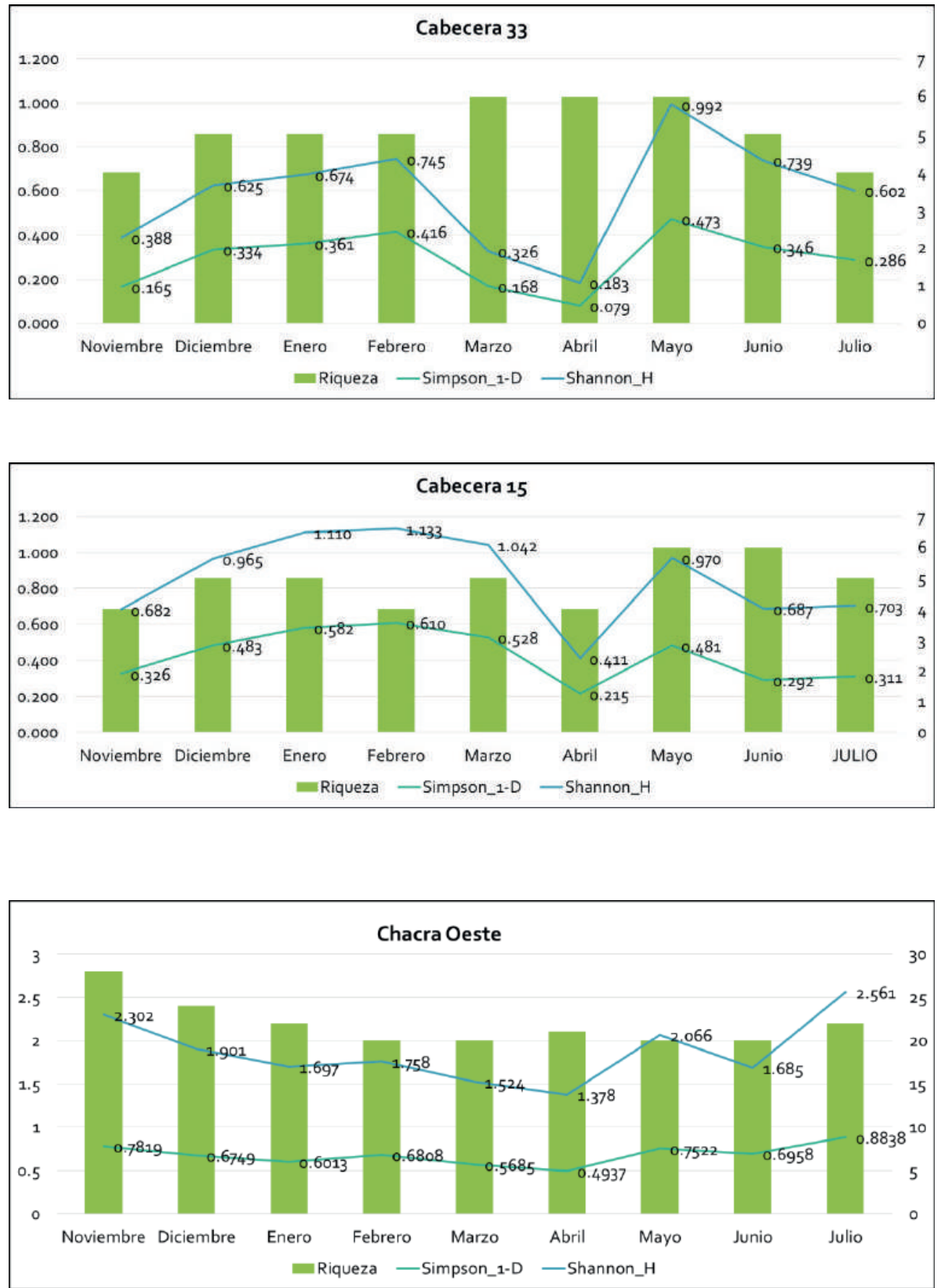

Figura 9. Variación de los índices de biodiversidad alfa en los meses de observación para los diferentes espacios evaluados en el AIJCH.

Como conclusiones de ésta investigación podemos indicar que: La composición de avifauna en el Aeropuerto Internacional Jorge Chávez (AIJCh) se basa en especies antrópicas y residentes del lugar, siendo L. pipixcan la única especie migratoria durante los meses de verano (diciembre -marzo). La zona de Chacra oeste, donde se encuentra una amplia cobertura vegetal es aquella que tiene mayor oferta de alimento, lugares de reposo, anidamiento y percha, en consecuencia, dicha zona presenta la mayor riqueza y abundancia. La temperatura 
ambiental es un factor que no condiciona la presencia estacional de las especies, sin embargo, la especie $C$. atratus incrementa su presencia en verano aprovechando las térmicas de aire caliente para realizar vuelos. El tamaño corporal, abundancia y tipo de vuelo de las aves fueron los factores que más influyeron en el riesgo de impacto aeronáutico, siendo $C$. atratus y la familia Laridae los que presentan mayor riesgo de impacto. El riesgo de impacto aeronáutico no está directamente relacionado con los índices de biodiversidad, sino más bien con la presencia de aves de gran porte y con vuelo de mayor altitud.

\section{REFERENCIAS BIBLIOGRÁFICAS}

Ayala-Pérez, V.; Arce, N. \& Carmona, R. 2013. Distribución espacio-temporal de aves acuáticas invernantes en la Ciénega de Tláhuac, planicie lacustre de Chalco, México. Revista Mexicana de Biodiversidad, 84: 327-337.

Barras, S.C. \& Dolbeer, R.A. 2000. Reporting bias in bird strikes at John F Kennedy International Airport, New York, 1979-1998. USDA National Wildlife Research Center-Staff Publications, 140p

Dukija, J. \& Gahlot, V. 2013. An evaluation of the effect of bird strikes on flight safety operations an international airports. International Journal for Traffic and Transport Engineering, 3: 16-33.

Bibby, C.; Jones, M. \& Marsden, S. 1998. Expedition field techniques: bird surveys. London: Expedition Advisory Centre, Royal Geographical Society; $134 \mathrm{p}$.

Gatto, A.; Quintana, F.; Yorio, P. \& Lisnizer, N. 2005 Abundancia y diversidad de aves acuáticas en un humedal marino del Golfo San Jorge, Argentina. El Hornero, 20: 141-152.

González, O. \& Málaga, E. 1997. Distribución de aves en el Valle de Majes, Arequipa, Perú. Ornitología Neotropical, 8: 57-69.

Guzmán, J.; Carmona, R.; Bojórquez, M. \& Palacios, E. 1994. Distribución temporal de aves acuáticas en el estero de San José del Cabo, BCS, México. Ciencias Marinas, 20: 63-103.

Küsters, E. 2000. Man-made wetlands and flight safety. In: International bird strike committee meeting,
25: 18. Amsterdam. IBSC 25 / WP SA1. 17-21 April 2000.

Leôncio, F.; Henrique, D.; De Paiva, B. \& Viera, L. 2010 Avifauna relacionada ao risco de colisóes aéreas no aeroporto internacional presidente Juscelino Kubitschek, Brasilia, distrito federal, Brasil. Revista Conexao SIPAER, 1:230-243.

Linnell, M.; Conover, M. \& Ohashi, T. 1999. Biases in bird strike statistics based on pilot reports. The Journal of Wildlife Management, 63: 997-1003.

Madrid, F.M. \& Elías, C. 2017 Avistamiento de aves en el campus de la universidad Ricardo Palma, Lima, Perú. Biotempo, 14: 167-177.

Marateo, G.; Grilli, P.; Ferretti, V. \& Bouzas, N. 2011. Diagnóstico de riesgo aviario en un aeródromo de un aérea megadiversa del Perú. Revista Conexao SIPAER, 3:203-227.

Meer, A.M. 2019. Bird-strike aircraft accidents and their prevention. Asian Journal of Science and Technology, 10: 9251-9257.

Mendonça, F.A.C. 2009. Gerenciamento do perigo aviário em aeroportos. Revista Conexão SIPAER, 1:153-174.

Ning, H. \& Chen, W. 2014. Bird strike risk evaluation at airports. Aircraft Engineering and Aerospace Technology: An International Journal, 86: 129137.

Novaes, W. \& Álvarez, M. 2010. Perigo aviário em aeroportos do nordeste do Brasil: análise das colisôes entre aves e aviôes entre os anos de 1985 e 2009. Revista Conexão SIPAER, 1: 47-68.

Owino, A.; Biwott. N. \& Amutete, G. 2004. Bird strike incidents involving Kenya Airways flights at three Kenyan airports, 1991-2001. African Journal of Ecology, 42:122-128.

Ralph, C.J.; Geupel, G.R.; Pyle, P.; Martin, T.E. \& De Sante, D.F. 2017. Riqueza de palomas y tórtolas (Columbidae) en el centro regional Universitario de Colón. Revista Colón Ciencias, Tecnología y Negocios, 4: 100-107.

Reca, A.; Úbeda, C. \& Grigera, D. 1994. Conservación de la fauna de tetrápodos. I. Un índice para su 
evaluación. Mastozoología Neotropical, 1:1728.

Remsen, J.V., Jr.; Areta, J.I.; Bonaccorso, E.; Claramunt, S.; Jaramillo, A.; Pacheco, J.F.; Ribas, C.; Robbins, M.B.; Stiles, F.G.; Stotz, D.F. \& Zimmer, K.J. 2019. A classification of the bird species of South America. American Ornithological Society. http://www.museum. lsu.edu/ $\sim$ Remsen/SACCBaseline.html

Schulenberg, T.; Stotz, D.; Lane, D.; O'Neill, J. \& Parker, III. T. 2010. Birds of Perú: Updated and revised Edition. Princeton University press. Serie Biodiversidad. CORBIDI.

Sodhi, N.S. 2002. Competition in the air: birds versus aircraft. The Auk, 119: 587-595.

Ugalde-Lezama, S.; Valdez-Hernández, J.I.; RamírezValverde, G.; Alcántara-Carbajal, J.L. \& Velázquez-Mendoza, J. 2009. Distribución vertical de aves en un bosque templado con diferentes niveles de perturbación. Madera y bosques, 15:5-26.
Vargas-Villegas, F. 2013. Informe técnico el peligro de fauna en los aeródromos del Perú. Callao: Corpac; p. 48. Report No.1.

Villegas-Patraca, R. \& Herrera-Alsina, L. 2015. Migration of Franklin's Gull (Leucophaeus pipixcan) and its variable annual risk from wind power facilities across the Tehuantepec Isthmus. Journal for Nature Conservation, 25: 72-76.

Washburn, B.E. \& Seamans, T.W. 2004 Management of vegetation to reduce wildlife hazards at airports. USDA National Wildlife Research Center-Staff Publications, 396p.

Xeno-canto. 2019. Base de datos para la identificación de sonidos. Colección de Sonidos AmbientalesInstituto Humboldt, Colombia. Naturalis Biodiversity Center (2015-2019). En: https://www.xeno-canto.org/contributor/ XMFDPACYJN?pg=44 leído el 20 de Agosto del 2019.

Received October 21, 2019.

Accepted December 3, 2019 